\title{
Uniportal Endoscopic Lateral to Medial Direction Transforaminal Lumbar Interbody Fusion: A Case Report and Technical Guide for Navigating Through Landmarks in Left Lumbar 4/5 Post Laminotomy Revision Lumbar Fusion Surgery
}

\author{
Pang Hung $\mathrm{Wu}^{1,2}$, Hyeun Sung Kim${ }^{1}$, Il-Tae Jang ${ }^{1}$ \\ ${ }^{1}$ Department of Neurosurgery, Nanoori Gangnam Hospital, Seoul, Spine Surgery, Seoul, Republic of Korea \\ ${ }^{2}$ Department of Orthopaedic Surgery, National University Health System, Jurong Health Campus, Singapore
}

Corresponding Author:

Hyeun Sung Kim, MD, PhD

Department of Neurosurgery, Nanoori

Hospital Gangnam, Seoul 731, Eonju-ro,

Gangnam-gu, Seoul, Republic of Korea

Tel: +82-2-6003-9767

Fax: +82-2-3445-9755

Email: neurospinekim@gmail.com

Received: September 28, 2020

Revised: November 11, 2020

Accepted: November 29, 2020
Post lumbar laminotomy anatomical changes can be disorienting to uniportal spinal endoscopist. This which led to many open conversionconversion to open surgery to verify landmarks. Isthmus tends not to be violated inis usually preserved in laminotomy and it can be used as a useful landmark for endoscopic fusion surgery. Unlike tubular microscopic surgery, endoscope possess more mobility; conveniently navigating through the identifiable anatomical landmarks in revision surgery with minimal fluroscopy. Uniportal Endoscopic Lateral to Medial Direction Transforaminal Lumbar Interbody Fusion, is a useful revision surgery technique with isthmus as reference point, it had not been described in literature. Case Presentation A 66 years old lady with two previous lumbar decompressive surgery to left $L 4 / 5$ presented with neurogenic claudication and instability. She was diagnosis was to have $L 4 / 5$ post-surgical spondylolisthesis with stenosis. She Informed consent was obtained consented for left $L 4 / 5$ revision uniportal endoscopic lateral to medial direction transforaminal lumbar interbody fusion, Endo (LM)-TLIF. Procedure started with drilling isthmus with from lateral to medial direction using exploratory bone drilling dissection technique to decompress and explore residual bony anatomical landmark. With proper definition of anatomical margins of intervertebral disc space, endoscopic guided discectomy, end plate preparation, cage and percutaneous pedicle screws insertion were done with aid of fluoroscopy and endoscopy to perform spinal fusion. Patient did well post operatively without any intraoperative complication.Conclusion Endo (LM)-TLIF is a viable, safe and efficacious method to explore a potentially challenging post-surgical ana- tomy around spinal canal in revision lumbar fusion

Key Words: Endoscopy, Endoscopic spine surgery, Lumbar fusion, Revision spine surgery, Spinal stenosis, Degenerative lumbar spondylosis

\section{INTRODUCTION}

Due to aging population, there is an increase incidence of degenerative spinal conditions which in turn leads towith corresponding increase increasing incidence of patients who had previously undergonesurgical history of previous spinal decompression". Progression of spinal stenosis and /or instability after lumbar decompression are some of the reasons for revision spinal fusion surgery on a previous spinal decompressed site ${ }^{3)}$. There is higher risk of of complications in revision surgery with some of the dreaded complications such as higher riskincidence of incidental durotomy, blood loss, wound break down due to devascularization from recurrent surgery ${ }^{2,3)}$. It is further complicated confounded by the various comorbidities of an elderly populations ${ }^{7)}$. All of these factors make revision spinal fusion a challenging surgery. Endoscopic spine surgery has several advantages which can be harnessed in provision of surgeryconsideration for revision surgery in for this group of post laminotomy and/or laminectomy patients requiring spinal fusion. In Endoscopic Spine Surgery, there is less soft tissue dissection, which minimizing minimized the damage to soft tissue and hence low- 
ered the chance of wound breakdown and blood lossi. A clear magnified view which allows careful dissection of scar tissue using endoscopic equipment. However, the disruption of the normal bony anatomical landmark from previous surgery and tissue scarring from previous surgery can be disorienting to the operating surgeon during endoscopic procedure. We would like to highlight a case example of a patient who had 2 previous decompression decompressive spinal surgery presenting with neurogenic claudication and spinal instability requiring spinal fusion. We perform a novel method of Uniportal Endoscopic Lateral To Medial Direction Transforaminal Lumbar Interbody Fusion;
Endo (LM) TLIF using exploratory bone drilling dissection approach which was not described in the literature, we discuss the various endoscopic fusion techniques and the advantage of Endo (LM) TLIF in revision spinal fusion surgery.

\section{CASE REPORT}

A 66 years old female patient who had no significant past medical history presented with left sided neurogenic claudication for 1 year. Claudication distance was less than $100 \mathrm{~m}$. Her leg pain
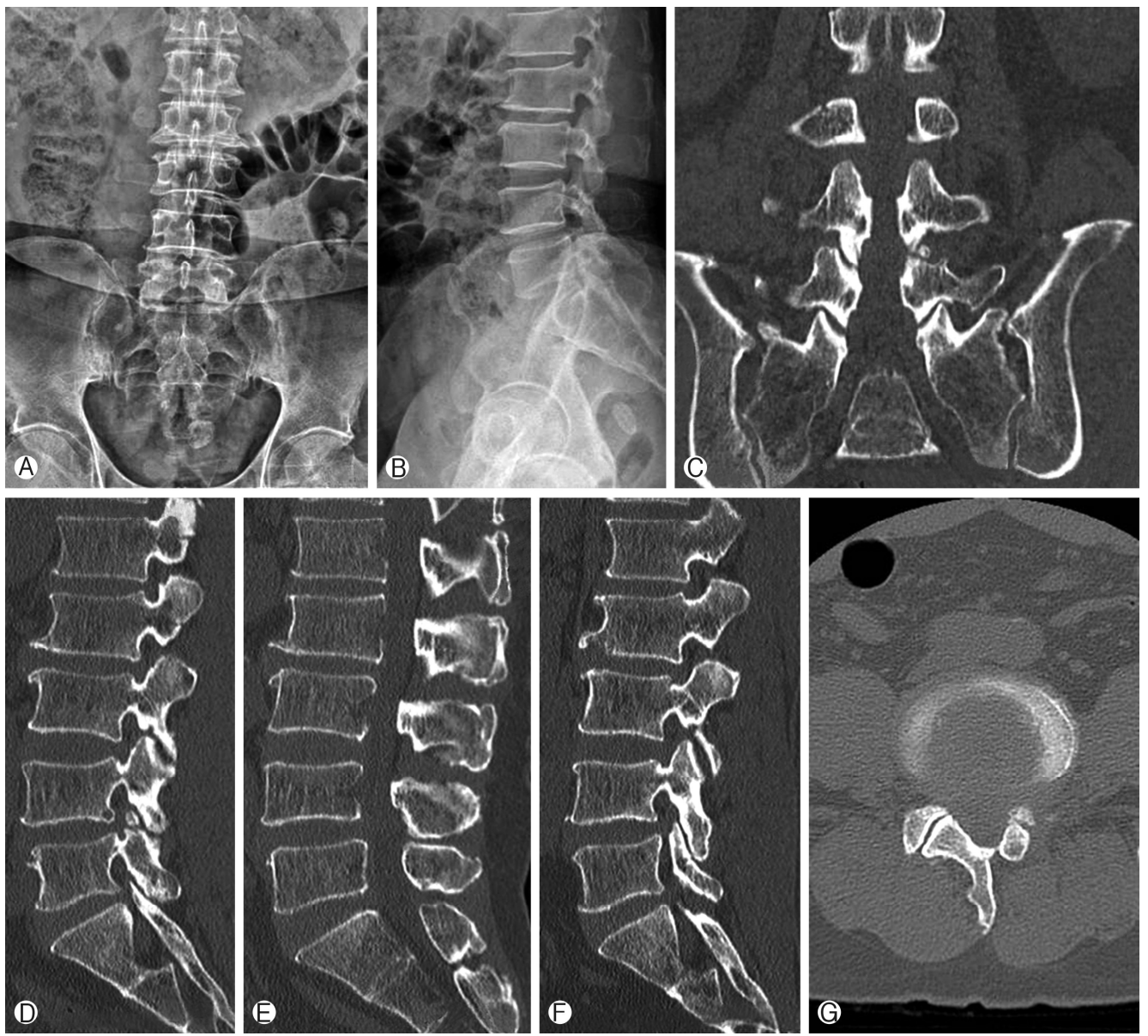

Figure 1. In this case demonstration, the 66 years old patient with left sided neurogenic claudication for 1 year with past history of 2 procedures: left L4/5 mini-open microscopic unilateral decompressive laminoforaminotomy and also left $L 4 / 5$ paraspinal approach foramina decompression done 4 years ago. X-ray and Computer Tomography scan is shown showing left $L 4 / 5$ grade 1 spondylolisthesis with superior articular process fracture and foramina narrowing. (A) and (B): Anteroposterior and lateral view showing decreased $L 4 / 5$ disc height and grade 1 spondylolisthesis. (C): Coronal CT view showing left $L 4 / 5$ facet disruption with a smaller size superior articular process fracture and inferior articular process decreased in size and shape. (D): CT left sagittal foramina view showing concordant findings with Coronal view with a smaller size superior articular process fracture and inferior articular process decreased in size, narrowing of left $L 4 / 5$ foramen by syndesmophyte of $L 4 / 5$ and superior articular process tip fracture fragment impingement is noted. $(E)$ and $(\mathbb{F}$ : Mid and left foramina sagittal view showing no significant central and contralateral foramina stenosis. (G): Axial $L 4 / 5$ Disc level cut showing, anatomical changes of left $L 4 / 5$ facet. In this case, previous resection of both inferior and superior articular process makes it unsafe to dock and drill on the intersection of superior articular facet and caudal lamina. An alternative start point at the lateral isthmus is more appropriate. 
visual analog scale (VAS) was $9 / 10$, she had lower back pain on changing position from sitting to standing and from lying to sitting, which suggested a component of spinal instability with a VAS score of 8/10. Her neurological examination was unremarkable. She had a past history of 2 procedures, a left L4/5 mini-open microscopic unilateral decompressive laminoforaminotomy and a left mini-open microscopic $L 4 / 5$ paraspinal approach foramina decompression done 4 years ago. In her preoperative workup, we checked her previous operative notes, she had no significant complications of previous surgery. Radiological investigations especially computer tomography with 3D reconstruction was done to assess post-operative changes to bony anatomy. Magnetic Resonance Imaging was done to evaluate area of spinal stenosis; any soft tissue compression such as prolapsed disc and facet cyst (Figure 1 and 2). Her Roentgenogram and computer tomography scan showed left L4/5 grade 1 spondylolisthesis with old superior articular process fracture and foramina narrowing (Figure 1). Both of which could be contributory to her current symptoms of spinal instability and neurogenic claudication. Her magnetic resonance imaging showed in paracentral sagittal view and axial view that she had left lateral recess stenosis at left $L 4 / 5$ with disc herniation in lateral recess (Figure 2). She had tried conservative treatment for past 1 year with no sign of improvement. She was consented for left L4/5 Uniportal Endoscopic Lateral To Medial Direction Transforaminal Lumbar Interbody Fusion; Endo (LM) TLIF. The goal of surgery was to decompress lateral recess and foramen of left $L 4 / 5$ with restoration of intervertebral disc height by interbody cage and fusion to provide spinal stability. The patient was well post-operatively, no perioperative complications, she was seen 6 months after surgery with VAS score of 1 in lower back and 0 in the leg, there was no longer any limitation of claudication.
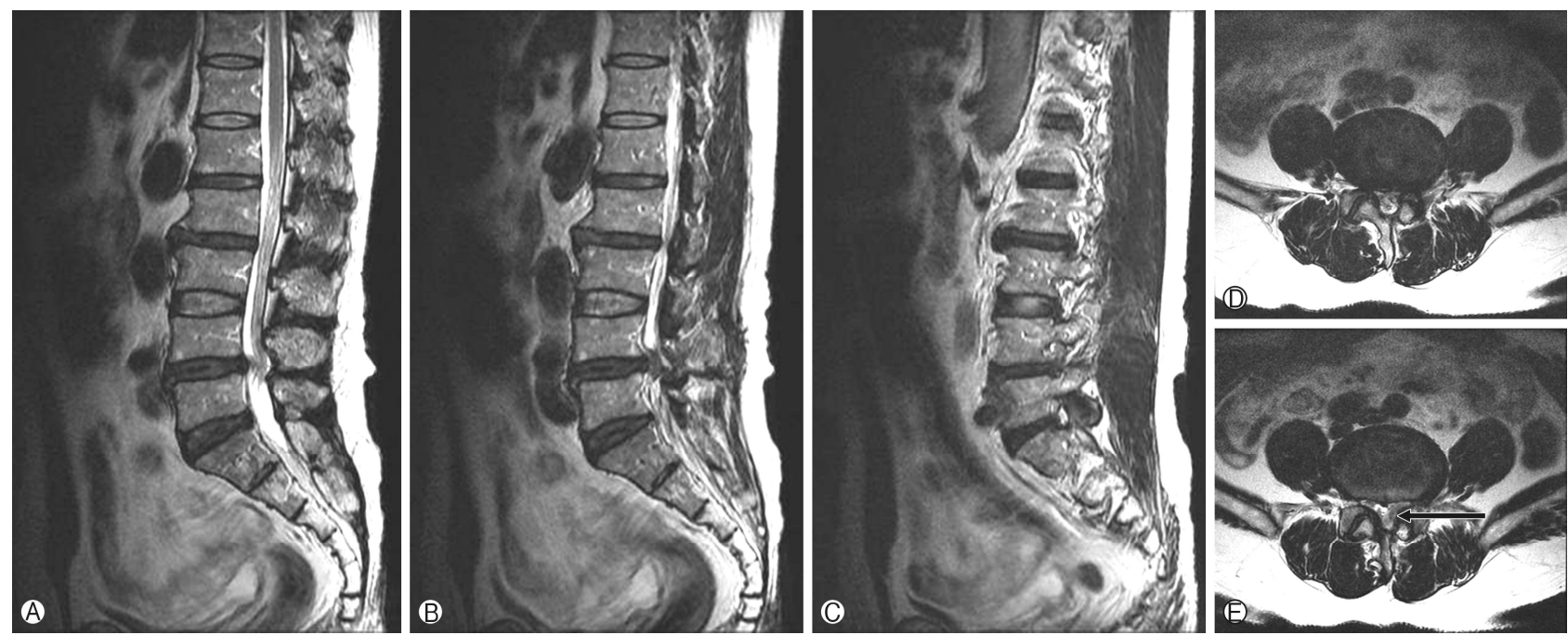

Figure 2. Magnetic Resonance Imaging of the same patient. (A): Mid sagittal cut showing no central stenosis, (B): Paracentral sagittal view showing left lateral recess stenosis at left $L 4 / 5$ with disc herniation in lateral recess. (C): Left foramina view showing left $L 4 / 5$ foramina stenosis. (D): Axial $L 4 / 5$ upper part of the disc level showing no central stenosis and dysmorphic facet. (E): Axial $L 4 / 5$ lower part of the disc level showing spinal stenosis and left paracentral disc herniation leading to compression of traversing nerve root show by red arrow. 
3). Both (1) and (2) shared a confusing similar description as transforaminal lumbar interbody fusion (TLIF). With improved equipment and techniques in endoscopic spine surgery ${ }^{8)}$, the authors adopted the Harms approach route for fusion using uniportal endoscopy with facet resection to create space for interbody cage insertion.

\section{Strength of Endoscopic Transforaminal Lumbar Lateral to Medial Lumbar Interbody Fusion}

In terms of open versus endoscopic transforaminal lumbar interbody fusion (TLIF) literature, there is paucity of literature on Uniportal Endoscopic TLIF, but evidence from Biportal TLIF surgery has been positive with better minimally invasive benefits and yet achieving as good outcome as open procedures ${ }^{5}$. In our experience uniportal endoscopic TLIF which explored the Endoscopic Exploratory bone drilling dissection is a technique which allows dissection of the soft tissue, bones and scars safely under endoscopic magnified vision. The unique feature of endoscopic procedure is the fact that the magnified view is directly close to within millimeters of the tissue being operated upon. Together with an endoscopiccoarse diamond burr which is light weight, one can carefully drill bone and soft tissue by layers safely. Continuous saline irrigation allows bone fragments which is drilled to be washed away from surgical field and flow out of the wound through the endoscope working channel. Once we get close to dura, radiofrequency and blunt probe can be used to tease off the scar from dura surface. Overall, the authors felt that with experience, this technique enhanced safety in fusion procedure especially in revision cases.

\section{Isthmus Versus Spinolaminar Approach for Inferior Facetectomy}

Traditional docking point In endoscopic interlaminar approach is at the junction of superior lateral aspect of caudal lamina and most medial inferior part of the facet joint called "V point" (Figure $3 \mathrm{C})^{6}$. However, morphological changes in anatomy of post-lami-
(A)
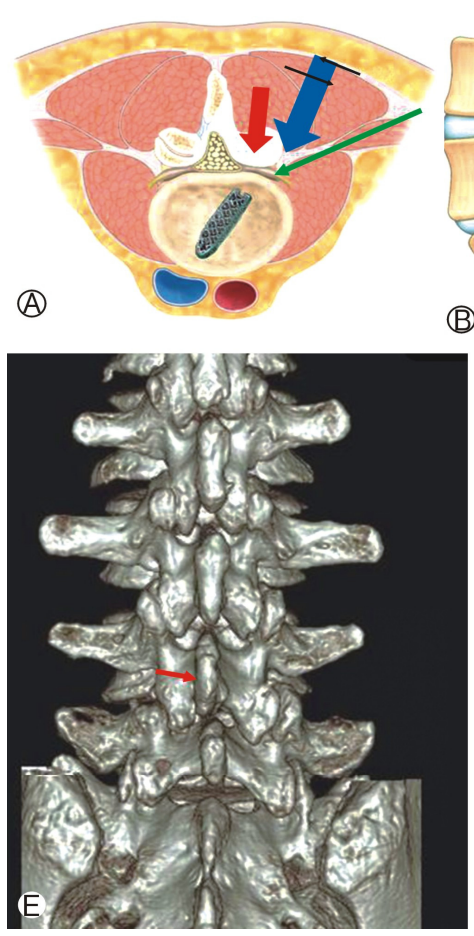
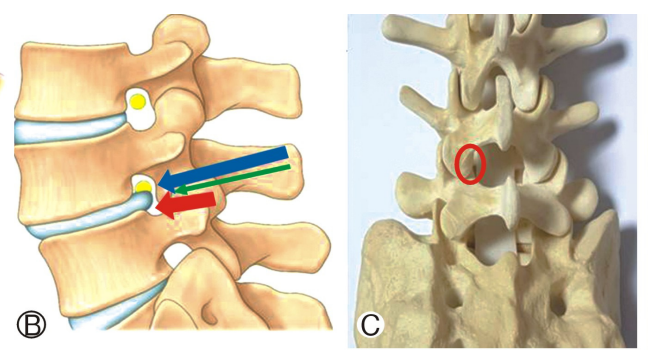
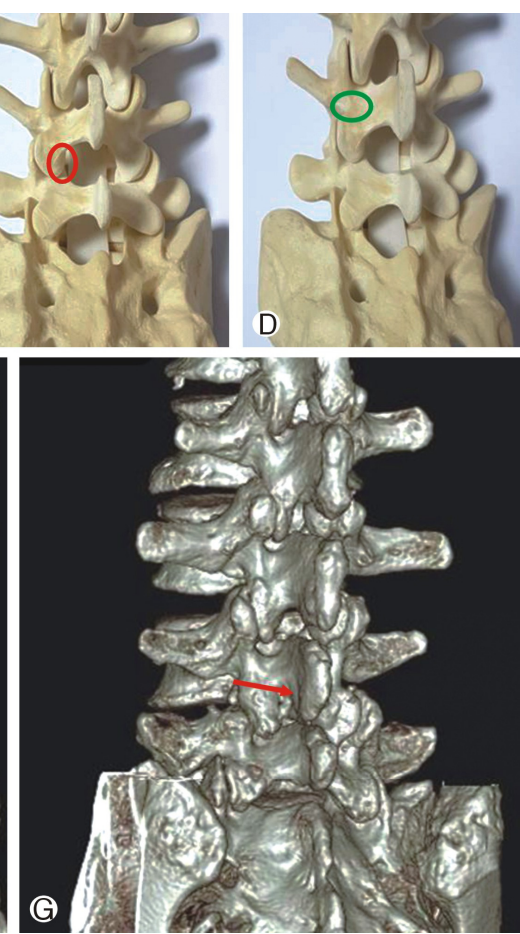

Figure 3. Various approach, docking and direction of exploratory bone drilling dissection for fusion. (A) and (B): Red arrow is direction of approach in posterior lumbar interbody fusion, blue arrow is approach in Harm's des cription of transforaminal lumbar interbody fusion, $\mathrm{ML}$ is medial to lateral direction of bony drilling on isthmus, usually deployed in uncomplicated uniportal endoscopic transforaminal lumbar interbody fusion by authors, LM is lateral to medial direction exploratory bone drilling dissection deployed in Endo (LM)TLIF, green arrow is trans Kambin facet sparing transforaminal lumbar interbody fusion. (C): The routine docking area of interlaminar approach and for uncomplicated uniportal endoscopic transfora- minal lumbar interbody fusion at intersection of superior lateral aspect of caudal lamina and medial inferior aspect of facet joint. (D): Isthmus docking for Endo (LM) TLIF. (E) to (G): 3D reconstructed CT scan showing detailed bony anatomy of the left L4/5 region, noting that there is decreased in size and shape of inferior articular and superior articular facet; isthmus and spinous process are spared which can useful landmarks in orientation in uniportal spinal endoscopy, red arrow showing direction of exploratory bone drilling dissection. 
notomy facet such as facet fracture, scar formation, facet subluxation and changes in facet morphology can be disorienting. Moreover in post laminotomy patients; medial half of facet joint was absent filling with scar tissue, dissection to identify medial facet could run the risk of incidental durotomy. Isthmus was preserved in decompression spine surgery ${ }^{14)}$ and hence it was a good landmark to refer to in revision fusion (Figure 3D). Overall, with all the above points considered, decision was made for Uniportal Endoscopic Lateral to Medial Direction Transforaminal Lumbar Interbody Fusion.

\section{Description of Endo (LM) TLIF}

Patient was positioned prone on a Wilson frame on a radiolucent table under epidural anesthesia with monitored sedation. Skin incision was over the midpoint of left $L 4$ pedicle on intraoperative image (Figure 4A).

\section{Docking, Working Channel Placement and Angle of Approach}

Isthmus was palpated and docked through blunt serial dilation with obtruators on left L4 Isthmus under fluoroscopic guidance. Finally we placed a $15^{\circ} / 1.37 \mathrm{~mm}$ outer diameter working channel at the isthmus and introduced interlaminar endoscope. We approach Isthmus at $45^{\circ}$ angle as per Harm's description of transfor- aminal lumbar interbody fusion ${ }^{4)}$ (Figure 4B).

\section{Exploratory Bone Drilling Dissection starting at Isth- mus for Inferior Articular Facetectomy}

Soft tissue dissection was performed exposing isthmus and lateral half of the facet (Figure $5 \mathrm{~A}$ ), we used a $3.5 \mathrm{~mm}$ coarse diamond endoscopic drill in a revision case. "Exploratory bone drilling dissection" was performed carefully with coarse diamond burr on isthmus in a lateral to medial direction from lateral aspect of isthmus to spinolaminar junction of cephalad lamina to expose underlying ligamentum flavum and the exiting nerve root underneath (Figure $5 B$ ). As this exploratory style of drilling was opposite of the routine Isthmus drilling from the medial to lateral direction with endoscopic burr after docking on the "V" point; the authors termed this as Endoscopic Lateral to Medial Direction Transforaminal Lumbar Interbody Fusion; Endo (LM) TLIF. Post-laminotomy adhesion was common cause of dura tear in revision surger $y^{13)}$ and the inferior articular facet was often stuck with adhesion making it difficult to retrieve en bloc with risk of dura tear. In this case, there was dense adhesion on the dura from scar tissue and inferior articular facet, hence, we drilled off inferior articular facet to gradually to bone dust. Endoscopic coarse diamond drill is relatively blunt and if one is careful in drilling layer by layer, it is a useful tool in dissecting both bone and soft tissue (Figure 4 and 5).
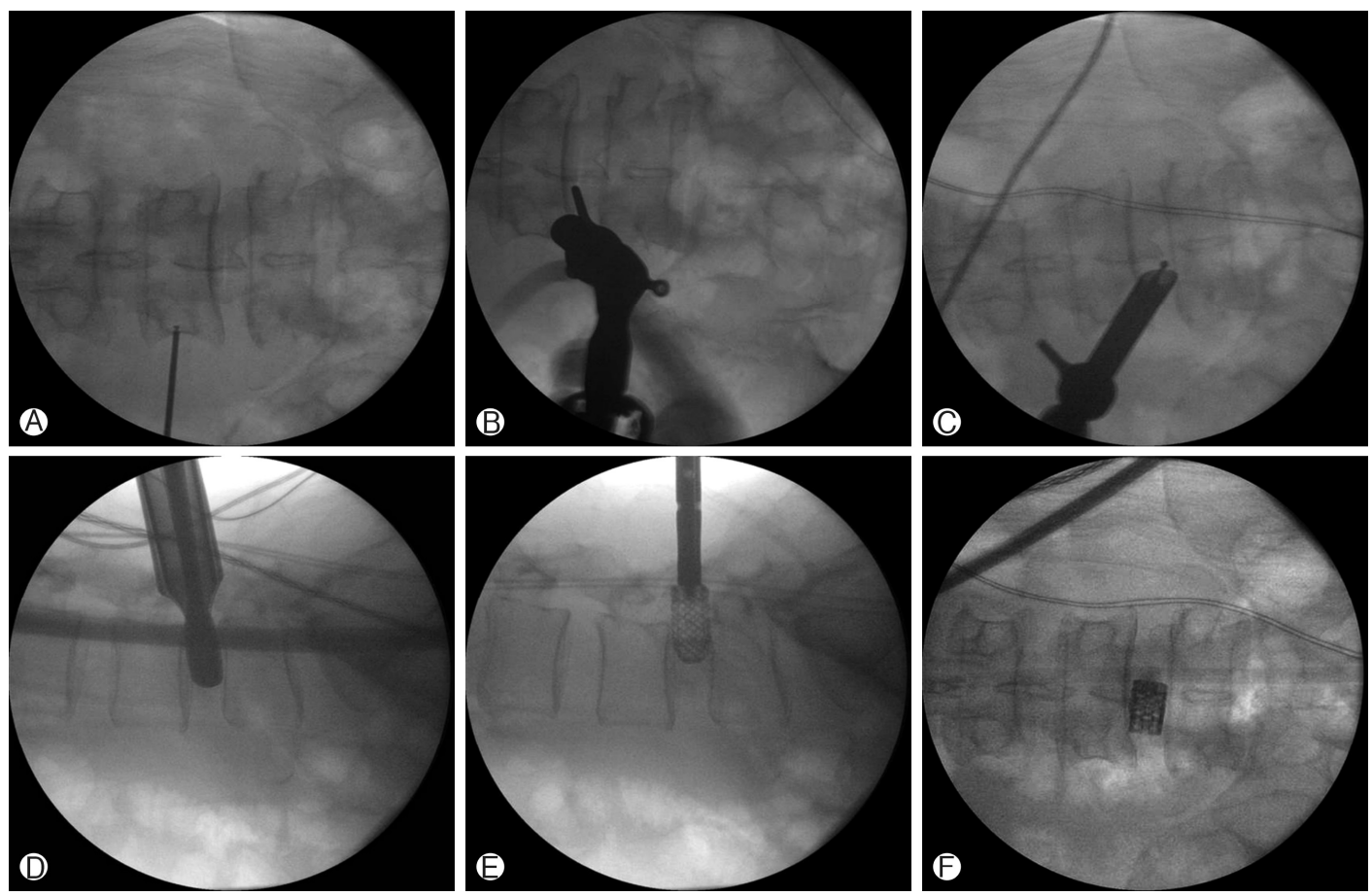

Figure 4. Intraoperative fluoroscopic images showing steps of Endo (LM) TLIF. (A): showing marking for skin incision over the left $L 4$ mid pedicle. (B): Docking of the endoscope over the left $L 4$ isthmus. (C): medial extent of exploratory bone drilling dissection ends at medial laminofacet junction. (D): With customized working channel, trial of size $12 \mathrm{~mm}$ cage inserted in the intervertebral space. (E) and $(\mathbb{F}$ : Size $12 \mathrm{~mm}$ height 3D printed titanium cage packed with allograft inserted in the left $L 4 / 5$ disc space. 


\section{Transverse Process Identification for Superior Articular Facetectomy}

The Junction of the transverse process and lateral superior articular process is a good landmark for exploratory bone drilling dissection from lateral to medial direction to remove the caudal superior articular facet. We used this landmark for drilling superior articular process to bone dust (Figure 5C).

\section{Ligamentum Flavum Resection and Exposure of Exiting and Traversing Nerve Root}

After resection of the entire ipsilateral facet, neural elements were seen after ligamentum flavum removed piecemeal by punches (Figure 5D). We changed to $30^{\circ} / 7.3 \mathrm{~mm}$ outer diameter endoscope to retrieve the herniated disc in lateral recess (Figure $5 E)$. If necessary we could have used the $30^{\circ}$ endoscope for contralateral decompression ${ }^{15)}$.

But since she had no significant foraminal stenosis, we did not do contralateral decompression in this case. After disc fragment retrieval, the larger working channel was re-deployed to evaluate neural elements and prepare end plates.

\section{Release of Adhesion, Discectomy and Plate Prepa- ration}

We rotated working channel with open bevel facing away from traversing nerve root to protect it from injury when we performed end plate preparation. The traversing nerve root was stuck with scar which was carefully released with radiofrequency probe, blunt forceps and probes. Radiofrequency probe, burr, blunt instruments and plasma coagulators were used for end plate preparation. Satisfactory endplate preparation shows mild punctate bleeding from adjacent end plates with cartilage denuded but anterior longitudinal ligament preserved.

\section{Cage Insertion}

The tip of working channel was impacted into the intervertebral disc space to protect the neural element and provided a guide for a trial of appropriate size cage. Generous amount of allografts were packed prior to inserting appropriate sized 3D printed size $10 \mathrm{~mm}$ height $\times 22 \mathrm{~mm}$ length, allograft packed titanium alloy cage through the working channel. We check intermittently with intraoperative imaging and endoscope to ensure no injury of the dura and cage was in optimal position (Figure 5F).

\section{Final Confirmation of Completion of Decompression}

Complete decompression showed signs of pulsating nerve roots and anemic nerve root turned pink. Drain was inserted

\section{Percutaneous Pedicle Screw Fixation}

We flattened the Wilson Frame and proceeded with fluoroscopic guided insertion of percutaneous pedicle screws with
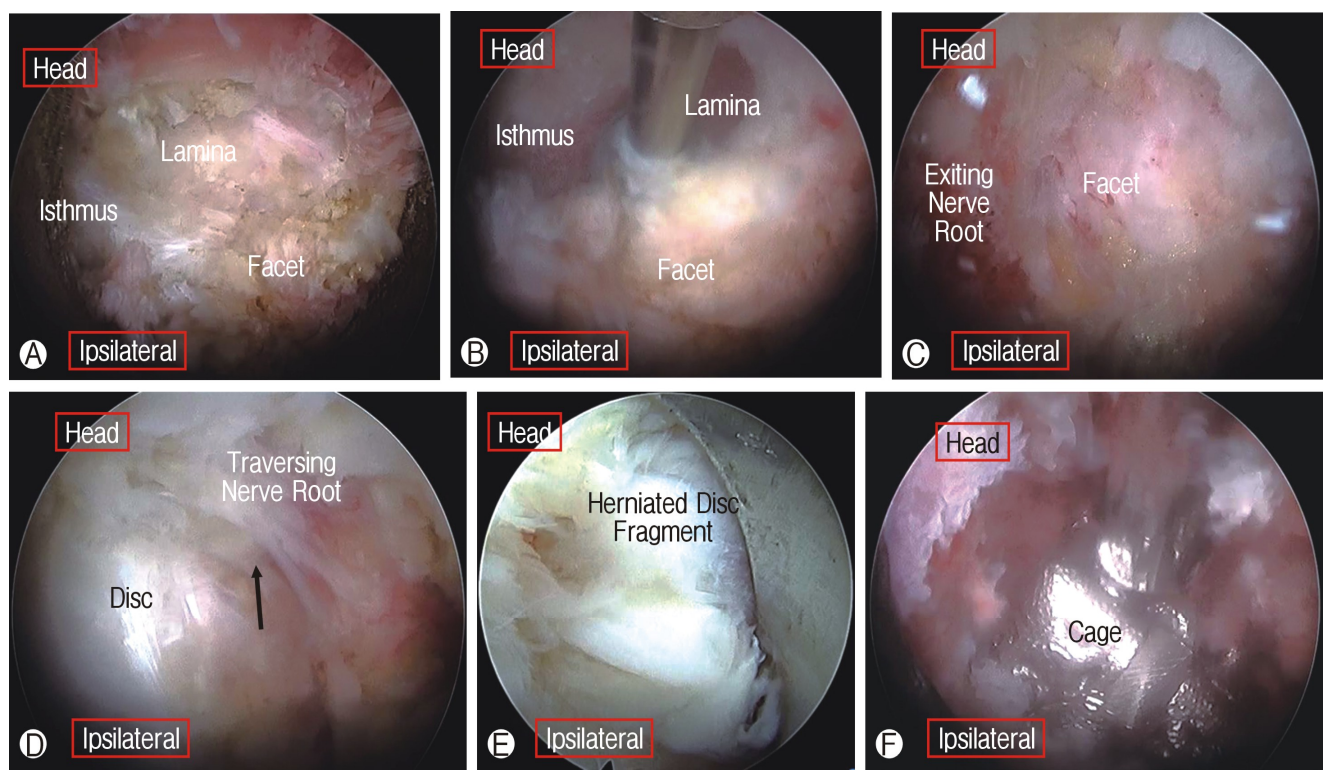

Figure 5. Intraoperative endoscopic pictures showing steps in Endo (LM) TLIF. (A): Exposure of isthmus, lamina and facet. (B): Lateral to medial exploratory bone drilling dissection to perform inferior articular facectectomy. (C): Exposure of exiting nerve root and superior articular facet. (D): Exposure of disc space and traversing nerve root. (E): We switched to smaller $30^{\circ} / 7.3 \mathrm{~mm}$ Outer diameter/171 $\mathrm{mm}$ length endoscope to perform discectomy. $\Theta$ : We switched back to $15^{\circ} / 1.37 \mathrm{~mm}$ Outer Diameter scope for end plate preparation, allograft bone grafting and cage insertion, cage position is adjusted under endoscopic visuali- zation. 
cement augmentations.

\section{Specific Perioperative Considerations}

Drain was removed on post-operative day one and the patient was mobilized on the next day as per the pain tolerated..

\section{Surgical Limitations}

There was limited amount of autogenous bone graft available was limited withobtained this method of fusion. Much of the bone was drilled to dust to determine landmarks in surgery and preventing inadvertent dura tear from forceful retrieval of bone fragment adhered to dura.

Allograft usage in this technique was preferred by the patient who opted not to have the morbidity of harvesting autograft from iliac crest as extra procedure. There was no statistical difference in terms of effects on fusion and outcome between allograft and autograft from iliac crest from low quality data".

The maximum inner diameter of working tube in various uniportal endoscopic companies are 10-11 mm. Large size customized working channel were needed to fit larger cages, in her case, a $10 \mathrm{~mm}$ cage was required

\section{Summary of 10 Key Technical Points of Surgery}

1) Preoperative imaging to evaluate anatomical changes prior to revision

2) Docking on the isthmus with blunt obturator under image guidance
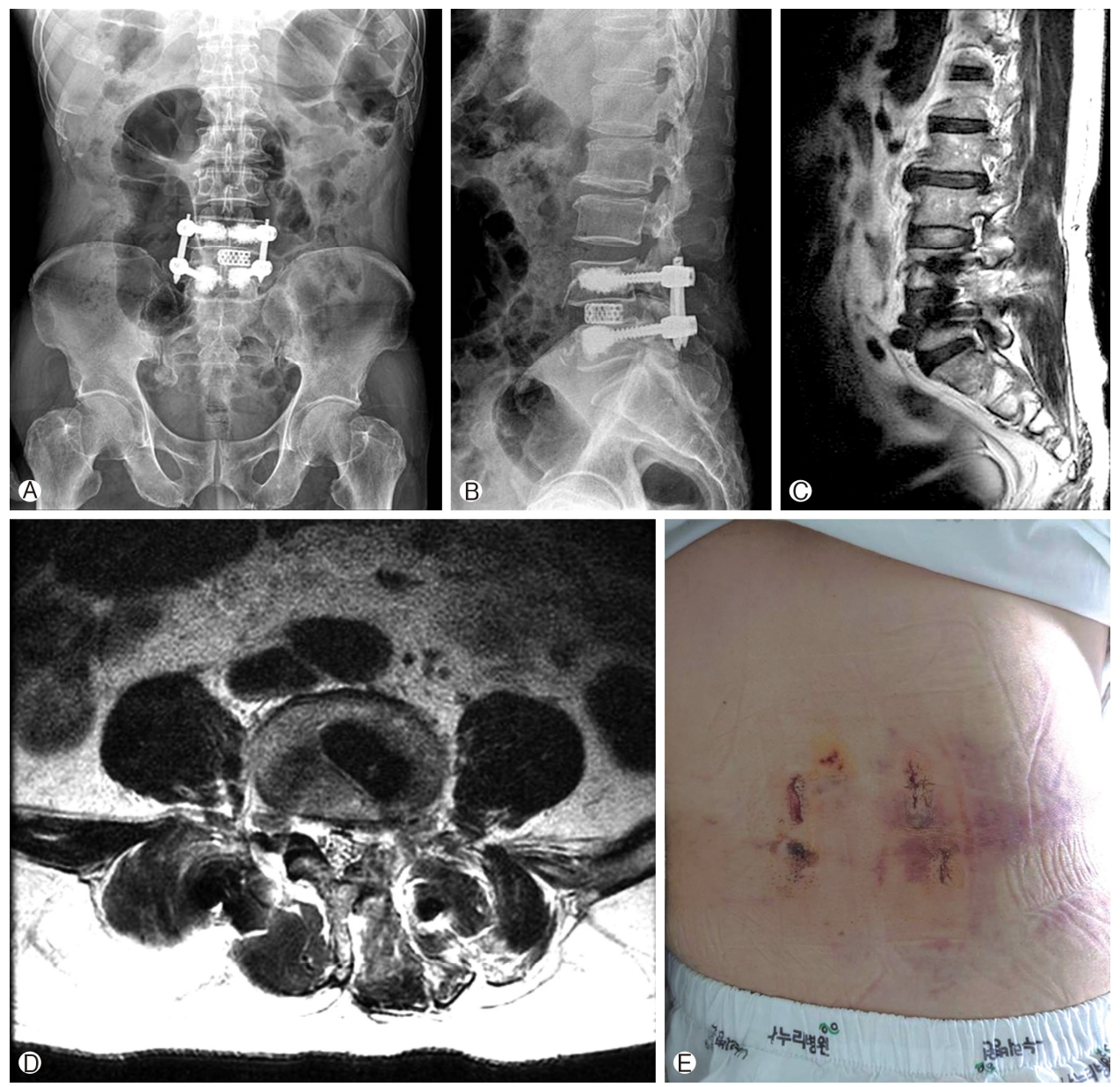

Figure 6. Post-operative imaging and skin incision on post-operative day 4 after drain removal. (A)(B): Anteroposterior and lateral view showing L4/5 Endo (LM) TLIF, with good height restoration, cage position and pedicle screw positions with cement augmentation. (C) and (D): MRI images of left foramina view and axial cut of L4/5 showing good, central, lateral recess and foramina decompression with minimal damage to surrounding soft tissue and muscle. (E): Picture of wound in post-operative day 4 prior to discharge, drain was removed on post-operative day 1 , showing mild acceptable amount of bruising good skin apposition, $3 \times 1 \mathrm{~cm}$ incision with $1.5 \mathrm{~cm}$ incision over the upper left L4 pedicle screw trajectory which is used concurrently by the working channel for Endo (LM) TLIF. 
3. Lateral to medial exploratory bone drilling dissection on isthmus close but superficial to exit nerve root with diamond burr for complete inferior facetectomy

4) Lateral to medial drilling of superior articular facet to expose and detach underlying ligamentum flavum

5) Low threshold to change a smaller diameter scope to perform discectomy and release adhesion

6) Rotate open bevel away from traversing nerve root during end plate preparation.

7) Optimal End plate preparation under direct endoscopic vision

8) Cage insertion using large endoscope working channel cannula

9) Release Wilson Frame to restore lordosis prior to screw fixation

10) Drain insertion to drain excess irrigation fluids and debris.

A case example shown (Figures and Video 1) showing revision left L4/5 Endo (LM) TLIF.

\section{Limitations of this Study Design}

As Uniportal Posterolateral Facet Sacrificing Endoscopic Transforaminal Interbody Fusion is a relatively new and evolving technique, the number of revision performed using this technique in our hospital and in the literature was limited to one case. This case report patient had a successful surgery and a good clinical outcome, but we need more numbers of patients and more long term data to review the safety and feasibility of the operation. Nevertheless, in our opinion $a$, this technique can be considered good technique consideration for revision lumbar spinal fusion.

\section{CONCLUSIONS}

Endo (LM) TLIF is a viable, safe and efficacious method to explore a potentially challenging post-surgical anatomy around spinal canal in revision lumbar fusion as an alternative approach in performing uniportal endoscopic spinal fusion and provides a viable, safe and efficacious method to explore a potentially challenging post-surgical anatomy around spinal canal in revision lumbar fusion.

\section{CONFLICT OF INTEREST}

Dr. HS Kim and Dr. PH Wu are editorial board members of the journal but were not involved in the peer reviewer selection, evaluation, or decision process of this article. There are no other potential conflicts of interest relevant to this article to declare.

\section{REFERENCES}

1. Buser Z, Brodke DS, Youssef JA, Meisel HJ, Myhre SL, Hashimoto R, et al.: Synthetic bone graft versus autograft or allograft for spinal fusion: A systematic review. J Neurosurg Spine 25: 509-516, 2016

2. Eichholz KM, Ryken TC: Complications of revision spinal surgery. Neurosurgical focus 15:E1-E4, 2003

3. Elgafy H, Olson D, Liu J, Lewis C, Semaan H: Effectiveness and safety of transforaminal lumbar interbody fusion in patients with previous laminectomy. Eur Spine J 24:810-816, 2015

4. Harms J: Dorsale Repositionsspondylodese bei lumbalen Spondylolisthesis: J. Harms zu J. Giel: In: Operat Orthop Traumatol 10:280-90, 1998 (Heft 4). Operative Orthopädie und Traumatologie 11:79-79, 1999

5. Heo DH, Son SK, Eum JH, Park CK: Fully endoscopic lumbar interbody fusion using a percutaneous unilateral biportal endoscopic technique: Technical note and preliminary clinical results. Biomed Res Int 43:E8, 2017

6. Ito F, Ito Z, Shibayama M, Nakamura S, Yamada M, Yoshimatu H, et al.: Step-by-Step Sublaminar Approach With a Newly-Designed Spinal Endoscope for Unilateral-Approach Bilateral Decompression in Spinal Stenosis. Neurospine 16:41-51, 2019

7. Kim JH, Kim HS, Kapoor A, Adsul N, Kim KJ, Choi SH, et al.: Feasibility of Full Endoscopic Spine Surgery in Patients Over the Age of 70 Years With Degenerative Lumbar Spine Disease. Neurospine 15:131-137, 2018

8. Kim M, Kim HS, Oh SW, Adsul NM, Singh R, Kashlan ON, et al.: Evolution of Spinal Endoscopic Surgery. Neurospine 16: 6-14, 2019

9. Lee CH, Chung CK: Health Care Burden of Spinal Diseases in the Republic of Korea: Analysis of a Nationwide Database From 2012 Through 2016. 15:66-76, 2018

10. Lin EY, Kuo YK, Kang YN: Effects of three common lumbar interbody fusion procedures for degenerative disc disease: A network meta-analysis of prospective studies. Int J Surg 60:224230, 2018

11. Morgenstern C, Yue JJ, Morgenstern R: Full Percutaneous Transforaminal Lumbar Interbody Fusion Using the Facet-sparing, TransKambin Approach. Clin Spine Surg 2019

12. Park JH, Roh SW: Long-term clinical and radiological outcomes following stand-alone PLIF surgery using expandable cylindrical threaded cages in patients with degenerative lumbar spine disease. Acta Neurochir (Wien) 153:1409-1416; discussion 1416, 2011

13. Smorgick Y, Baker KC, Herkowitz H, Montgomery D, Badve SA, Bachison C, et al.: Predisposing factors for dural tear in patients undergoing lumbar spine surgery. J Neurosurg Spine 22:483486, 2015

14. Spetzger U, Bertalanffy H, Naujokat C, von Keyserlingk DG, Gilsbach JM: Unilateral laminotomy for bilateral decompression of lumbar spinal stenosis. Part I: Anatomical and surgical considerations. Acta Neurochir (Wien) 139:392-396, 1997

15. Wu PH, Kim HS, Jang I-T: How I do it? Uniportal full endoscopic contralateral approach for lumbar foraminal stenosis with double crush syndrome. Acta Neurochirurgica 162:305-310, 2020 